\title{
Anesthetic Considerations in Jansen's Metaphyseal Chondrodysplasia: A Case Report of a Pediatric Difficult Intubation in a Rare Type of Dwarfism
}

\section{Jorge-Reynolds L M, Meyerson P, Rodriguez L I, Del Rio I, Mavarez A C* Department of Anesthesiology, University of Miami Miller School of Medicine, USA}

*Corresponding author: Ana C Mavarez, M.D., Department of Anesthesiology, Perioperative Medicine and Pain Management, University of Miami Miller School of Medicine 1611 NW 12th Ave, Ste C-300, Miami, FL, USA 33136, Tel: 786-556-0198; Email: ana.mavarez@jhsmiami.org

\section{Case Report \\ Volume 4 Issue 4}

Received Date: November 11, 2019

Published Date: December 11, 2019

DOI: $10.23880 /$ accmj-16000166

\section{Abstract}

Metaphyseal Chondrodysplasia Type Jansen (MChTJ) is a very rare autosomal dominant skeletal disorder characterized by abnormal cartilage formation in the metaphysis, leading to progressive growth plate abnormalities and a characteristic short limb dwarfism. Skull and facial deformities, renal impairment, hypercalcemia, and hypophosphatemia are seen in most of these patients. We report a case of a 6-year-old male child with diagnosed MChTJ undergoing multiple teeth extraction under general anesthesia. Our intent is to elucidate possible problems that may be encountered in the anesthesia management of patients with MChTJ and discuss the periopertive measures to take when these arise.

Keywords: Jansen's metaphyseal chondrodysplasia; General anesthesia: Difficult intubation; Congenital abnormalities; Dwarfism

Abbreviations: MChTJ: Metaphyseal Chondrodysplasia Type Jansen; PTH: Parathyroid Hormone; OMFS: Oral Maxillofacial Surgery; ETT: Endotracheal Tube.

\section{Introduction}

Metaphyseal chondrodysplasia Type Jansen (MChT), is an extremely rare and serious disorder with autosomal dominant pattern that affects long bones, leading to a characteristic short limb dwarfism by impairing growth plate development, parathyroid hormone (PTH)independent hypercalcemia and hypophosphatemia. It is caused by a sporadic mutation of the parathyroid hormone receptor 1 (PTHR1) and PTH related peptide [13]. Approximately 20 cases of MChTJ have been recorded in the medical literature. Because of the limited evidence with few case reports, and almost non-existent description of the anesthetic management of these patients, the anesthesiologist must be vigilant to the possible anatomical and physiologic abnormalities. Skull and facial bone irregularities can lead to difficult 


\section{Anaesthesia \& Critical Care Medicine Journal}

ventilation and endotracheal intubation [4]. Electrolyte abnormalities are common and can lead to cardiac arrhythmias and postoperative respiratory dysfunction. Musculoskeletal deformities may cause difficulty with venous access and positioning; and renal dysfunction can alter the metabolism and excretion of the anesthetic medications given [2]. The purpose of this case report is to describe successful anesthetic management of a child with MChTJ who required multiple dental extractions under general anesthesia.

\section{Case presentation}

A 6-year-old male boy $(17.7 \mathrm{~kg}$ weight and $96 \mathrm{~cm}$ height), with diagnosed metaphyseal chondrodysplasia Jansen type (MChTJ) presented to the emergency room with gingival abscess surrounding broken tooth in lower left bicuspid and multiple teeth caries and breaks. He was previously evaluated by two community pediatric dentists who were unwilling to perform extractions given his complex skeletal dysplasia and airway abnormalities. After no significant improvement of symptoms with oral antibiotic coverage, the parents decided to bring the patient to our institution. Patient was admitted and evaluated by oral maxillofacial surgery (OMFS) who decided to perform dental extractions with drainage of peri-gingival abscess under general anesthesia.

Previous medical history included premature birth at 36 weeks gestation via elective cesarean delivery given nuchal umbilical cord. On prenatal ultrasound there was evidence of bilateral femur bowing and abnormal rib cage. Work up in the neonatal intensive care unit for congenital metabolic bone disease revealed a diagnosis of MChTJ when he was 3 months old by genetics. He had no allergies, previous surgeries, or fractures. Patient had a normal behavior for his age with no intellectual disability but mild developmental delay. He was home-schooled and living with both parents and two siblings. There was no family history of any skeletal dysplasia.

Patient was admitted one day before surgery. Preoperative evaluation by pediatric anesthesiologist showed small stature (height was $96 \mathrm{~cm}$, less than the third percentile for his age with a Z score of -4.57), shortening of the upper and lower limbs, bowing of both legs, frontal bossing, prominent eyes, narrowing of the thoracic chest wall, normal spine, clubbing and multiple contracture of joints and waddling gate. Airway examination revealed appropriate mouth opening with Mallampati IV classification, micro-retrognathia, flat, low, and narrow hard palate, multiple loose upper and lower teeth, thyromental distance (TMD) less than 3 fingerbreadth and limited neck extension.

Laboratory values showed hypercalcemia of $11.4 \mathrm{mg} / \mathrm{dl}$ (normal values $8-11 \mathrm{mg} / \mathrm{dl}$ ) with low PTH of $9.45 \mathrm{pg} / \mathrm{ml}(15-65 \mathrm{pg} / \mathrm{ml})$, hyperkalemia of $5.6 \mathrm{mEq} / \mathrm{L}$ $(3.4$ - $4.7 \mathrm{mmol} / \mathrm{L})$ with no changes in the electrocardiogram (ECG), hypophosphatemia of $3.6 \mathrm{mg} / \mathrm{dl}$ (3.8 $-5.9 \mathrm{mg} / \mathrm{dl}$ ) and elevated alkaline phosphatase to 673 units/L (117 - 357 units/L). Cervical spine radiographs reported misalignment of the odontoid process and second cervical vertebral body. Bone scan showed increased density at the base of the skull with underdeveloped sinuses, shortening of ribs, rachitic changes, generalized lytic changes with severe decrease mineralization and irregular appearances of metaphysis throughout the skeleton.

A multidisciplinary team evaluation of the patient prior to surgery included, pediatric orthopedic surgery who recommended careful endotracheal intubation with minimal cervical spine manipulation; endocrinology to correct his electrolyte abnormalities and optimize him for surgery; cardiology which recommended a 2D echocardiogram that revealed patent foramen ovale and mild peripheral pulmonary stenosis which were normal variants for his age, and genetics was on board perioperatively due to his syndrome. Due to concerning cervical instability, potential risk of acute cervical bone fractures and possible difficult endotracheal intubation, the plan was to secure the airway performing an awake nasotracheal fiberoptic intubation (NFOI) prior to induction of general anesthesia to avoid manipulation of the neck and to enhance oral access for tooth extraction. The patient was premedicated with $2 \mathrm{mg}$ of midazolam and $0.1 \mathrm{mg}$ of glycopyrrolate given via preexisting 22gauge peripheral intravenous (IV) line in the arm. The child was taken to the operating room and after placement of standard monitors, intranasal oxymetazoline spray was applied for local vasoconstriction. Adequate sedation was provided with three sequential doses of ketamine IV at $0.5 \mathrm{mg} / \mathrm{kg}$ were given for a total of $25 \mathrm{mg}$. Previously lubricated nasopharyngeal airway 20 French size was inserted to right nare without complications. He maintained spontaneous ventilation during nasal dilation. After three minutes, nasal airway was removed.

NFOI was attempted with a $4.5 \mathrm{~mm}$ cuffed nasal rae endotracheal tube (ETT) but could not pass through the nare because it was too large. Unfortunately, a smaller $4.0 \mathrm{~mm}$ cuffed ETT would not load into the smallest 


\section{Anaesthesia \& Critical Care Medicine Journal}

pediatric video fiberoptic scope available. Decision was made to start inhalational induction of anesthesia with incremental sevoflurane via facemask, followed by an IV propofol bolus of $30 \mathrm{mg}$. Mask ventilation was easily accomplished without an oral airway. Once the patient was adequately anesthetized, video laryngoscopy was performed with a MAC blade size 2.5 using manual in-line stabilization, a grade $2 \mathrm{a}$ view was attained and a $4.5 \mathrm{~mm}$ cuffed ETT was inserted successfully on first attempt. The ETT was properly secured and the patient underwent an uneventful dental extraction surgery that lasted approximately 10 minutes.

No neuromuscular blocker drug was used during the anesthesia, 12.5mg of IV fentanyl were given during surgery and for postoperative nausea and vomiting prophylaxis $1.5 \mathrm{mg}$ of IV ondansetron were given before extubation. He was emerged of anesthesia uneventfully and was successfully extubated awake and following commands without any complications. Anesthesia time was approximately one-hour total. He was transferred to the recovery room on right lateral decubitus position and in stable condition with blood pressure of $120 / 56 \mathrm{mmHg}$, heart rate of 120 , respiratory rate of 20 and 36.6 degrees of temperature. Patient did not show signs of airway edema, stridor, or croup; had no nausea or vomiting episodes and for pain control $265 \mathrm{mg}$ of IV acetaminophen were given. He remained in the recovery room for 1.5 hours and then moved back to the ward where he was later discharged home seven hours later, that same day uneventfully.

\section{Discussion}

MChTJ is a rare form of short limb dwarfism, originally described by Murk Jansen in 1934 [5]. It is characterized by impaired growth plate development, asymptomatic hypercalcemia and hypophosphatemia [6] with low or undetectable levels of PTH. Inherited in an autosomal dominant pattern [7] the majority of cases are the result of sporadic mutations of the PTHR1, resulting in a constitutively active PTH1R that is normally active in its monomeric form. Four missense mutations in the PTH1R gene have been identified in affected, but unrelated patients with the syndrome: H223R, I458R, T410P and T410R 8, with H223R mutation being the most frequent. The receptor is abundant on the surface of osteoblasts, mainly within the Metaphyseal growth plate and cells of the kidneys. Most cases appear to be caused by de novo mutations, and only few parent-to-child transmissions of disease causing PTH1R mutation have been reported [3]. MChTJ signs are present at birth and is usually diagnosed during early childhood, based on a combination of radiographic and biochemical abnormalities. MChTJ presents clinically with several facial abnormalities such as a receding chin or retrognathia, micrognathia, highly arched palate, wide cranial sutures and ocular hypertelorism [7]. Other clinical findings include small lower rib cage and fractured ribs, "bell-shaped" thorax, widen costochondral junctions, abnormal pelvis, bowing of the legs, short limb dwarfism, clubbed fingers with short hands and diminished muscle mass and swelling around the hips and knees [7,8]. As individuals age, they can also demonstrate limited flexion as affected joints become stiffer, a waddling gait as certain joints become fixed in a permanent flexed position [7]. Intellectual disability and psychomotor retardation have not been reported in affected individuals with MChTJ [8]. The present case shows the classical clinical abnormalities of MChTJ.

The diagnostic radiographic manifestations include severe metaphyseal involvement of all the tubular bones, large epiphyses with relative normal bone structure and short, mildly bowed diaphysis. A wide space between the epiphyses and metaphysis is a characteristic abnormality seen in the tubular bones of the hands, feet as well of the extremities [9].

The anesthesia management of patients with MChTJ should include a complete preoperative anesthesia evaluation with particular attention to head, neck and airway examination, assessment of musculoskeletal deformities of extremities and spine; as well as, cardiovascular, renal and endocrine abnormalities. These patients may represent a challenge to pediatric anesthesiologists because of potential difficult airway, electrolyte aberrancies and anesthetic medication effects attributable to potential and cardiac and renal dysfunction. A multidisciplinary team approach should be involved in the preoperative care and planning of patients with rare and complex diseases, ensuring medical optimization before surgery and careful intraoperative planning from choosing the adequate type of anesthetic whether it be a general anesthetic or regional and airway.

Due to facial deformities including micro-retrognathia described in MChTJ cases, bag-mask ventilation and endotracheal intubation could be difficult. Takkunen, et al. [10] reported an adult patient with MChTJ followed since birth that was found to have severe tracheobronchomalacia that merit surgical tracheobronchial reinforcement prior to scoliosis correction surgery [10]. Physical examination of the 


\section{Anaesthesia \& Critical Care Medicine Journal}

airway remains paramount for developing a plan for airway management. Preoperative planning for difficult intubations is essential. Nevertheless, contingency plans must always be considered. Access to alternative airway equipment and developing backup plans enhance patient safety. Children with difficult intubations are associated with high risk of complications such as temporary hypoxemia, bradycardia and cardiac arrest [11]. Fiadjoe et al described that such complications are associated with more than two intubation attempts, weight less than $10 \mathrm{~kg}$, short TMD, and three direct laryngoscopy attempts before using an indirect intubation technique [11].

Careful evaluation of the spine needs to be done prior to the patient being intubated. Cervical spine imaging preoperatory should be considered in patients with MChTJ specially if there is neck immobility, pain, or neurologic deficits. In-line immobilization with two providers during intubation or fiberoptic intubation is safe techniques in these cases [11]. Khan et al [4] reported a case of MChTJ with severe involvement of the superior cervical spine, although the patient had no neurologic findings or cervical spine instability.

Careful positioning of patients with MChTJ is critical during the anesthesia care, as their limb deformities might increase the risk of peripheral nerve injuries given extremity malposition causing stretch or compression, or pressure sores caused by ischemic pressure with reduction in perfusion of small areas. The anesthesiologist must facilitate safe positioning with adequate padding to prevent these injuries [12]. Joint abnormalities may also cause difficulty in obtaining venous access in these patients as contractures may cause anatomy distortion.

MChTJ is typically associated with severe, and generally, asymptomatic early-onset hypercalcemia and hypophosphatemia, with low-normal or undetectable serum PTH levels. Hence, most MChTJ patients develop biochemical features suggestive of hyperparathyroidism, including elevated serum calcium, low serum phosphorus and elevated alkaline phosphatase activity, but without elevated PTH levels [3].

Patients with electrolyte disorders are known to be a challenge for an anesthesiologist because of vital system involvements. Hypercalcemia, although usually asymptomatic, may be associated with hypercalciuria, nephrocalcinosis and nephrolithiasis [12] renal dysfunction, polyuria and dehydration. ECG evaluation in the preoperatory period is recommended as Hypercalcemia is associated with increased risk of arrythmias and hypertension [13]. Although, long term exposure to Hypercalcemia was not found to increase cardiovascular risk in a patient with effective control of hypercalciuria with oral bisphosphonates and thiazide diuretics [2]. Patients with MChTJ should be admitted prior to the day of surgery for adequate hydration and ensuring euvolemia and laboratory evaluation for further preoperatory optimization.

Hypophosphatemia can cause muscle weakness and hyporreflexia that may be present in patients with MChTJ. Hypophosphatemia patients had been reported to have respiratory problems, especially in the postoperative period [14]. The possibility of prolonged mechanical ventilation in patients with hypophosphatemia can be anticipated. Neuromuscular function should be monitored carefully when neuromuscular blocker drugs are given during anesthesia as muscular dysfunction can arise even with apparent complete reversal. Slow phosphorus replacement over 6-8 hours must take place to avoid the complications of rapid infusion which are hypocalcaemia and formation of calcium phosphate calcifications [15]. Urgent surgeries in a patient with hypophosphatemia should alert the anesthesiologist to the possibility of multiorgan failure and perhaps the need for postoperative mechanical ventilation.

Other electrolyte abnormality less commonly seen in patients with MChTJ is hyperkalemia that carries risk especially in terms of anesthesia because it can have specific symptoms on the nerves, muscles and gastrointestinal tract but the most typical symptom is on the cardiovascular system [14]. Preoperative ECG is important to evaluate for arrythmias. Anesthetic drugs including paralytics like succinylcholine and anesthesia maneuvers like hypoventilation may worsen hyperkalemia. The decision to proceed with a scheduled surgical procedure in a patient with hyperkalemia can be very challenging, it can be optimized preoperatively and if is an emergency case it is usually decided by a mutual agreement between surgery team and anesthesia provider [16]. Intraoperative management strategies can include intravenous administration of calcium, sodium bicarbonate, insulin with or without glucose, inhalation of albuterol and hyperventilation.

We wanted to draw attention the anesthetic considerations that an anesthesiologist should have in patients with MChTJ. Due to the complexities and potential risks associated with this genetic defect, patients with MChTJ may not be candidates for ambulatory surgical centers or in office dental procedures where resources are limited. Referal to an inpatient 


\section{Anaesthesia \& Critical Care Medicine Journal}

pediatric facility is highly recommended. Micrognathia and cervical joint abnormalities should be evaluated preoperatively because of possibility of difficult intubation. Hypercalcemia, hyperkalemia and hypophosphatemia should be corrected preoperatively for a properly functioning of the cardiac and respiratory systems. Postoperative observation will depend on the type and complexity of the surgery but early discharge is possible if meeting all discharge criteria from the post anesthesia recovery unit.

\section{References}

1. Shimomura-Kuroki J, Farooq M, Sekimoto T, Amizuka N, Shimomura Y (2017). Characterization of a PTH1R missense mutation responsible for Jansen type metaphyseal chondrodysplasia. Odontology 105(2): 150-154.

2. Onuchic L, Ferraz-de-Souza B, Mendonca BB, Correa PH, Martin R (2012) Potential effects of alendronate on fibroblast growth factor 23 levels and effective control of hypercalciuria in an adult with Jansen's metaphyseal chondrodysplasia. The Journal of Clinical Endocrinology \& Metabolism 97(4): 10981103.

3. Nampoothiri S, Fernandez-Rebollo E, Yesodharan D, Gardella T J, Rush E T, et al. (2016) Jansen Metaphyseal Chondrodysplasia due to Heterozygous H223R-PTH1R Mutations With or Without Overt Hypercalcemia. The Journal of clinical endocrinology and metabolism 101(11): 4283-4289.

4. Khan R, Oakes P, Fisahn C, Burgess B, Kirkpatrick K M, et al. (2017) Skull Base and Cervical Spine Involvement in Jansen Syndrome: Case Report. Pediatric Neurosurgery 52(2): 140-143.

5. Jansen M (1934) Über atypische chondrodystrophie (achondroplasie) und über eine noch nicht beschriebene angeborene wachstumsstörung des knochensystems: metaphysäre dysostosis. Zeitschr Orthop Chir 61: 253-286.

6. Minagawa M, Arakawa K, Takeuchi S, Minamitani K, Yasuda T, et al. (2008) Jansen-type Metaphyseal Chondrodysplasia: Analysis of PTH/PTH-related Protein Receptor Messenger RNA by the Reverse Transcriptase-Polymerase Chain Method. Endocrine Journal 44(4): 493-499.
7. Schipani E. Jansen Type Metaphyseal Chondrodysplasia (2015) Retrieved from NORD: National Organization for Rare Disorders web page: https://rarediseases.org/rare-diseases/jansen-typemetaphyseal-chondrodysplasia/

8. Silve C, Juppner H (2005) Jansen's metaphyseal chondrodysplasia. Retreived from Orphanet encyclopedia online.

9. Campbell J B, Kozlowski K, Lejman T, Sulko J (2000). Jansen type of spondylometaphyseal dysplasia. Skeletal Radiology 29(4): 239-242.

10. Takkunen O, Cozanitis D, Halttunen P, Kaitila I (1986). Tracheobronchomalacia in an adult with metaphyseal chondrodysplasia. Annales Francaises d'anesthesie et de Reanimation 5(5): 527-529.

11. Fiadjoe J E, Nishisaki A, Jagannathan N, Hunyady A I, Greenberg R S, et al. (2016) Airway management complications in children with difficult tracheal intubation from the Pediatric Difficult Intubation (PeDI) registry: a prospective cohort analysis. The Lancet Respiratory Medicine 4(1): 37-48.

12. Knight D J W, Mahajan R P (2004). Patient positioning in anaesthesia. BJA Education 4(5): 160-163.

13. Kessel D, Hall C M, Shaw D G (1992). Two unusual cases of nephrocalcinosis in infancy. Pediatric Radiology 22(6): 470471.

14. Goktas U, Tekin M, Kati I (2016). Anesthesia management of Jansen's metaphyseal dysplasia. Eastern Journal of Medicine 21(1): 52-53.

15. Ianov I, Wilkerson D (2010) Hypophosphatemia and acute postoperative respiratory distress. J Ark Med Soc 106(11): 265-266.

16. Huang L, Yarl W, Liu H (2019). Patient with Hyperkalemia for Surgery: Proceed or Postpone? Translational Perioperative and Pain Medicine 6 (1): 17-19. 